\title{
THE CIDOC CRM GAME: A Serious Game Approach to Ontology Learning
}

\author{
Anais Guillem ${ }^{\mathrm{a}}$, George Bruseker ${ }^{\mathrm{b}}$ \\ ${ }^{\text {a }}$ HIVE lab, UC Merced, Merced, United States - aguillem@ucmerced.edu \\ ${ }^{a}$ FORTH, - Centre for Cultural Informatics, Heraklion, Greece - bruseker@ics.forth.gr
}

\author{
Commission VI, WG VI/4
}

\begin{abstract}
KEY WORDS: CIDOC CRM, Serious Game, Formal Ontology, pedagogy, Heritage Information Integration
\end{abstract}
\begin{abstract}
:
Formal ontologies such as CIDOC CRM (Conceptual Reference Model) form part of the central strategy for the medium and longterm integration of cultural heritage data to allow for its greater valorization and dissemination. Despite this, uptake of CIDOC CRM at the ground level of Cultural Heriage $(\mathrm{CH})$ practice is limited. Part of the reason behind this lack of uptake lies in the fact that ontologies are considered too complicated and abstract for application in real life scenarios. This paper presents the rationale behind and the design of a CIDOC CRM game, the intent of which is to provide a learning mechanism to allow learners of wide backgrounds and interests to approach CIDOC CRM in a hands-on and interactive fashion. The CIDOC CRM game consist of decks of cards and game boards that allow players to engage with the concepts of a formal ontology in relation to real data in an entertaining and informative way. It is argued that the CIDOC CRM Game can form an important part of introducing the basic elements of formal ontology and this standard to a wider audience in order to aid wider understanding and adoption of the same.
\end{abstract}

\section{INTRODUCTION}

To the average cultural heritage professional who has come into contact with the concepts of 'formal ontology' or 'CIDOC CRM', the next concepts that would come to mind might not be 'game' or 'fun'. To the contrary, while the enormous potential of CIDOC CRM has even led it to be recognized as an ISO standard for cultural heritage, a reputation for complexity and abstraction accompanies this and other formal ontologies such that many specialists are reluctant to approach the subject. This gap between cultural heritage researchers and a practical knowledge of the use of formal ontologies for representing structured data is a genuine loss for research. While formal ontologies like CIDOC CRM hold the ambition to provide the long term solution to data heterogeneity problems (Doerr and Iorizzo, 2008; Smith, 2003), their widespread implementation can only be carried out if there is a bottom up appropriation of the standard by cultural heritage institutions and professionals in the field. There is an irony that while formal ontologies have specifically been designed on an empirical basis to solve a practical problem of data integration (Doerr, 2003) and have strong high level introductions (Doerr, Ore, Stead, 2007), research into the equally practical problem of how to introduce these ontologies in a hands-on and accessible manner to noncomputer science specialized users and cultural heritage professionals has not become a major area of research. This has left these highly practical tools with an undeserved reputation for being impractical. We end up then with a robust standard, excellent specialized research and development in the field, but a problem in properly scaling up understanding of the standard to a wider audience.

Part of meeting this challenge involves creating teaching/learning tools that can effectively communicate both the benefits and the practical use of an ontology to users with no specialist interest in ontologies as such. At this first dissemination and appropriation level, the ontology should be communicable and teachable in such a way that it can be taken up as if learning something as fun as a game. Creating this first access level will enable audiences as diverse as domain experts, management and the interested public to gain a first serviceable understanding of an ontology and how to practically implement it.

Within the context of this goal, this paper explores a project to elaborate a CIDOC CRM game as a teaching tool. The aim of this teaching tool is to fit into the area of introducing, popularizing and giving a direct idea of what a formal ontology is, what its basic concepts are and what it can do. The game thus aims to enable the first activities of discovery of the ontology and can be used in progressively more complex scenarios in order to allow teaching and learning CIDOC CRM in an engaged and interactive manner. It provides a unique approach to introducing an ontology, eschewing a traditional lecture and presentation method in favour of directly involving learners in appropriating the concepts and applying them with their own problem solving skills.

In what follows, we will present our analysis of some of the main problems facing the dissemination of formal ontologies such as CIDOC CRM, present the card game solution proposed to meet part of this challenge and outline the results of introducing this game in real workshop scenarios.

\section{STATE OF THE ART}

\subsection{The Problem}

The problem of teaching formal ontologies has to do with what Cooper (1999) called 'cognitive friction'. That is to say, while a user would ultimately benefit from mastering the skill, the upfront cost appears so high as to discourage uptake. There are a number of distinct properties of formal ontologies that contribute to this perception as being intellectual, distant and hard to approach that form the key problems to be faced in devising means to introduce ontologies in a friendly and approachable way.

Some of the main factors include:

The roots of formal ontology in the disciplines of philosophy and information sciences ground it in a long intellectual lineage of reasoning and logic (Guarino 1998; Smith and Welty, 2001). This is an advantage for creating robust data models, but can 
create significant communication barriers with those approaching the topic from outside traditions of philosophy and information sciences.

Another factor that can cause an adoption barrier is the manner in which formal ontologies generalize and criticize domain terminology. Precisely because of its need to serve as an integrating information tool, formal ontologies generalize from input data structures and as a result propose classes of a higher, more general nature than those commonly suggested in a bespoke or subject specific data structure (Doerr, 2003). This can generate scepticism from the domain specialists whose categories are being abstracted (Sure et al., 2009). It is important for any teaching tool to be able to demonstrate how the ontology can re-express data from a source without losing meaning.

The introduction of formal ontologies also means the introduction of an abstract and novel way of representing data, which is a serious challenge to teach. The task of connecting abstract classes to instances/relations that they are meant to describe poses particular issues. Because of the high level definitions offered in formal ontologies this task can be extremely difficult to imagine without examples and, particularly, examples relative to one's domain of expertise. Further, the representation of metadata in terms of a graph structure is a novel form of representation for most learners of an ontology, the manner and use of which needs to be clearly communicated. There is a need to convey both how relations are restricted for use between different classes and also how they can be deployed in series to create complex meaningful multipart statements out of initially flat data structures.

The above barriers can be overcome but to do so, for parts of the audience not predisposed to logical puzzles, requires new ways of exposing this information that engages the nonspecialist learner via not only visual but also tactile, oral and group learning. A means of making the learning task hands-on and interactive opens the opportunity for a genuine engagement with and hence appropriation of the ideas at hand by the learner. By not only hearing a lecture on the concepts of a formal ontology concepts but also having a chance to interact with them in a direct manner, learners can link the ideas to the knowledge that they already have. When the learning audience can face the above questions not in the abstract but as they would occur in concrete scenarios within their domain of expertise, then the functionality and usefulness of an ontology can be concretely demonstrated and its logical divisions and its argumentation and logic assimilated.

\subsection{CIDOC CRM}

CIDOC CRM forms an interesting case of a robust standard that offers genuine possibilities to a community but must bridge a knowledge gap between conceptual modellers and domain users. Data heterogeneity is native to cultural heritage studies and is non-reducible as a factor thereof. The study and management of cultural heritage and the information that we learn and produce about it is an inherently multidisciplinary process. Thus CIDOC CRM, which offers a neutral semantic frame in which to re-express data in a common, but nonprescriptive, format offers high potential functionality to cultural heritage specialists and institutions (Doerr, 2009). Yet though CIDOC CRM has been under development since the mid-1990s, its uptake among the broader community of cultural heritage professionals and practitioners cannot be said to be high owing, at least in part, to its reputation for complexity and abstraction as detailed above.

The irony in this situation is that while formal ontology sounds abstract, it is in fact a profoundly practical solution to the tangible problem of managing precisely the data overload that cultural heritage professionals face. Furthermore, unlike custom made technical solutions which may, in the short term, aid in solving such problems, but in the long term increase cultural heritage professional's dependence on IT experts and IT budgets, formal ontologies are an information technology designed to be understandable and applicable by a domain expert and thus to create data which is long term sustainable and reusable by $\mathrm{CH}$ institutions. CIDOC CRM encoded data are technology independent, giving a means by which the cultural heritage institution or expert can understand and control the process by which their information is formatted and interpreted. There is therefore a strong, if poorly understood incentive for cultural heritage institutions to adopt such systems in order to protect their long-term interest and investment in information (Oldman et al., 2014).

There is certainly uptake of the standard both in specialist projects as demonstrated in ever more numerous conference papers, and in marquee projects such as the work done at the British Museum in the ResearchSpace project (http://www.researchspace.org/) and the National History Museum of Germany in Nuremberg' Wiss-ki project (http://wiss-ki.eu/), and European projects such as Parthenos (http://www.parthenos-project.eu/) inter alia. That being said, the widespread take up of the standard at a ground level is still not to be observed. Despite interest, there is bottleneck to adoption. The formal ontology approach can only work when domain experts learn and can apply a formal ontology standard in order to re-express their data in a lingua franca (Doerr and Crofts, 1999) (Doerr and Crofts, 1999) Like any language then, a formal ontology requires training programmes, dissemination strategies and community harmonization/verification practices. The ground activity required, however, is training.

While there is a wide array of highly valuable training material already available from the official CIDOC CRM site, the material is expressed in a chiefly visual and non-interactive manner. The potential user base of the ontology, however, would seem to require meeting the needs of those whose interests and specializations vary with regards to their objects and subjects not to mention roles such as management, research, conservation, general interest and so on. If a path to appropriation of the standard is to be built for such a wide audience, then a teaching tool which facilitates surpassing the learning barriers to ontologies outlined above remains to be created.

\section{THE CIDOC CRM GAME AS AN ONTOLOGY LEARNING TOOL}

\subsection{Aim and Principles}

To address, at least in part, this pedagogic challenge, we set out to conceive a teaching tool that could be used either individually or in a class setting by a total beginner to the CIDOC CRM and which would allow the learner to immediately engage with, apply and understand the ontology and its concepts relative to their area of knowledge. The conceptual structure of a card game lends itself as a teaching tool because of its straightforwardness, ease of implementation and immediate familiarity and materiality. The context of a card game is 
immediately recognizable to users of all levels. The materialization of an ontology as a set of cards renders it visible and tactically available. The competitive nature of card games takes psychological focus off the complexity and the abstraction of the task at hand as an intellectual exercise and refocuses players on the gaming process. Finally, the medium of cards allows the addition of extra symbolic system and visual clues to the raw ontology in order to make it more immediately understandable, easing the appropriation of the abstraction of class and relation concepts.

There are also a number of features characteristic to a formal ontology that the medium of representation on cards can help introduce and clarify. First, ontologies are not an intellectual tool that one learns in a linear fashion. It is not in the best interest of the learner to pick up an ontology and read it from cover to cover. Rather, ontologies are learned in parts and through patterns. The medium of cards allows the user to approach these parts directly. Another aspect of the medium of cards which is useful in introducing an ontology is the random and sometimes serendipitous nature of sorting through the materialized classes and relations. Sorting through a deck of cards allows one to come across classes and relations that one might not have found in scanning through a list or running a search in a digital environment. Finding such new relations can cause the learner to re-evaluate their initial conceptions and thereby understand the ontology better. Finally, ontologies are logical representations of scientifically agreed ways of reasoning about a field of discourse. These generalized forms may not at first seem familiar to a learner of the standard. In designing example scenarios that encourage reasoning over these particular problems and representing them in CIDOC $\mathrm{CRM}$, the cards create a learning environment which encourages users to critically engage and understand the modelling decisions represented in the ontology.

\subsection{Game Materials}

The basic game materials designed were: three decks of cards, a CRM manual, a game scenario, and an expandable set of playing boards. The three decks of cards, designed to mimic regular playing cards, are used to represent respectively: the CRM classes, the CRM relations and a deck of instance cards relative to a specific scenario.
3.2.1 The CRM Class Cards: These cards have the size of regular playing cards. On the back side, they are identifiable by the repeated CRM blue logo pattern. The front side consists of two information panels. The upper panel gives the class label in a colored panel that also displays a distinct symbol to indicate the class' relative location in the CRM hierarchy.

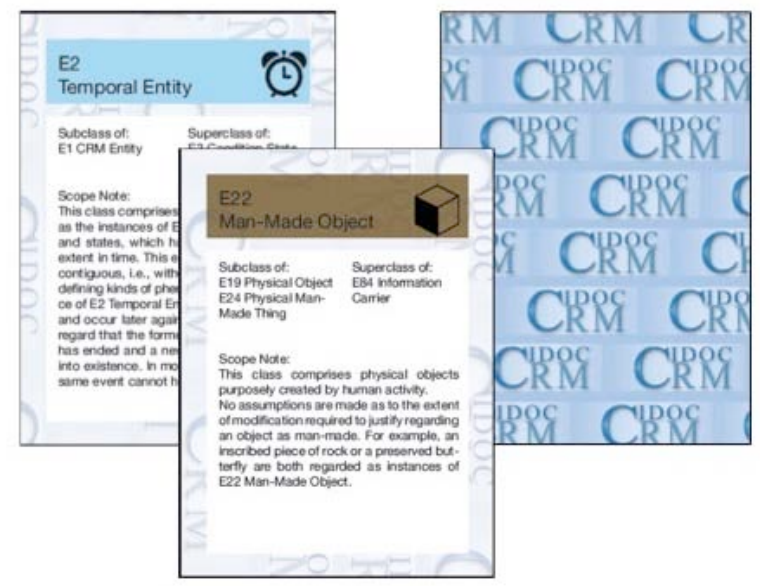

Figure 1. CRM Class Cards

The large, central panel takes up the main part of the card and indicates both the superclasses and subclasses of the class as well as its scope note. Where the scope note is too long, the note is truncated to its first section. The cards cannot replace the CRM manual for a full understanding of the classes, but they already give a much quicker immediate clue or hint as to what the class is for. This first encounter aims to encourage the learner to go further and check the complete information in the manual as necessary.

3.2.2 The CRM Relation Cards: These cards are half the size of normal playing cards. They are given this size to make it easy to work with them to visually connect classes. There is one main section of the card in which are indicated the property label, as well as the domain and the range for which the relation can be used. In order to help the learner/player, the sides of the cards are marked with colors that give a clue as to the type of class cards for which a relation could be used. The design choice of making the cards smaller eliminates the possibility of giving the scope notes for the relations that the cards represent. This tradeoff is worth it, however, in terms of game playability and the visual representation of a graph model through the card game.

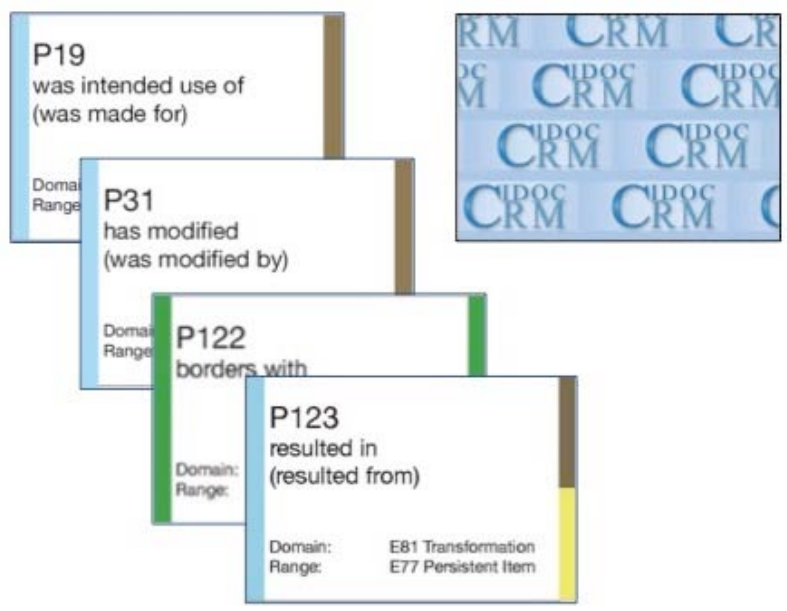




\section{Figure 2. CRM Relation Cards}

These two decks and the CRM manual independently already provide a tool for hands on appropriation of the CIDOC CRM ontology. The decks allow one to quickly sort through the various classes and relations declared by CRM and understand them in relation to each other.

In order to make a learning game, however, a further deck of cards was designed to provide instances from a sample scenario for which CRM classes and relations would be used to model the hypothetical data scenario.

3.2.3 The Instance Cards: Depending on the audience being presented to, a deck of cards containing example instances from a real data scenario, ideally highlighting potentially difficult conceptual classes and relations, is generated. These cards have the same dimension as the class cards, but are differentiated by a different coloured backing. The cards are given a title in the top panel which indicates their semantic value. An illustration in the main panel further gives visual clues as to the type of information being documented. Finally, an experimental section on the card was created to play with the idea of assigning more or less difficulty level to different concepts.

In the initial version of the game, the instance cards displayed data units related to the Initial Training Network-Digital Cultural Heritage (http://www.itn-dch.eu/) study of the Byzantine church Asinou located in the Troodos mountains of Cyprus. This scenario was selected because of its immediate relevance to the interdisciplinary group of researchers involved in that project, who were the test subjects of the game scenario. The case-study of Asinou was well known to the participants each from their respective disciplinary perspective.

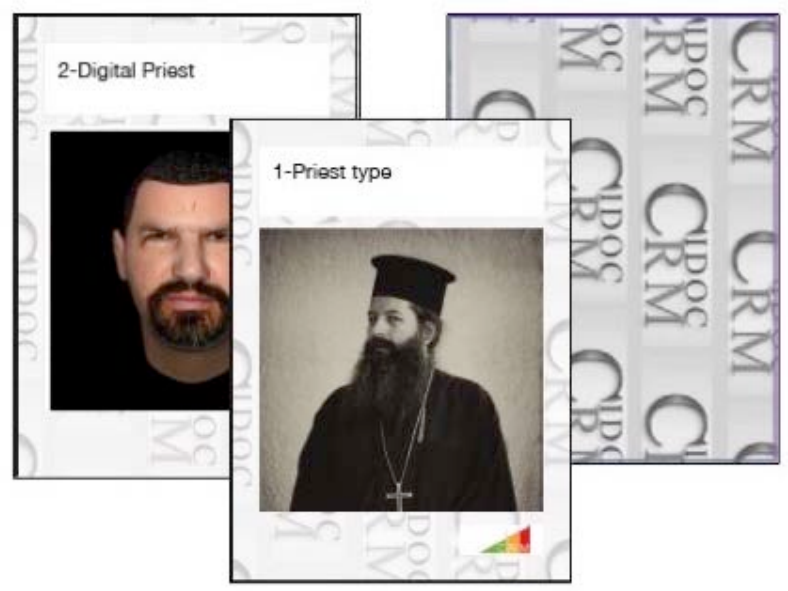

Figure 3. Instance Cards

Since then further example sets have been generated for other user scenarios. The instances represented were selected in order to demonstrate the heterogeneity of data that can be modelled under CIDOC CRM, allowing the connection of otherwise disparate seeming practices and research results.
3.2.4 The Semantic Game Boards: The initial version of the game was played without boards, which very quickly revealed the need to add this element. The Subject-Verb-Object structure of semantic data structures was not initially obvious to nonspecialist users. The introduction of a simple game board representing this structure helped facilitate game-play greatly. The introduction of an add-on game board was conceived to allow unlimited connections among data and helps replicate the environment of a semantic data graph.'

\subsection{Game Design}

3.3.1 The colour and iconographic scheme: As mentioned above, one of the advantages of materializing the ontology as a set of cards is that it allows building visual clues for appropriating the ontology that would not be appropriate within the context of the standard itself. Particularly, the size of the ontology often presents itself as a learning challenge to users. It is not clear to new learners of CIDOC CRM where to begin with the ontology and how to understand it as a whole.

As an event based ontology, however, with several clear high level distinctions in the ontology, it is possible to partition the model into a high level pattern of events in which objects, physical or conceptual, come and go, actors participate as agents, and to which time and place can be attributed. Further, to any entity any number of names or classifications can be applied. This high level intellectual schema, already elaborated by the CIDOC CRM SIG, can be re-represented through a division of the classes into respective colours and iconographic codes.

\begin{tabular}{|c|c|c|c|}
\hline Colour & Symbol & $\begin{array}{l}\text { Class Type } \\
\text { Represented }\end{array}$ & $\begin{array}{l}\text { Class } \\
\text { Root }\end{array}$ \\
\hline & & $\begin{array}{l}\text { Temporal } \\
\text { Classes }\end{array}$ & E4 \\
\hline & & $\begin{array}{l}\text { Conceptual } \\
\text { Classes }\end{array}$ & E28 \\
\hline & & $\begin{array}{l}\text { Physical } \\
\text { Classes }\end{array}$ & E18 \\
\hline & & Actor Classes & E39 \\
\hline & & Place Classes & E53 \\
\hline & $\begin{array}{cccc} & T & T & T \\
Y & 1 & 1 & y \\
* & * & * & * \\
i & 1 & 1 & i\end{array}$ & Type Classes & E55 \\
\hline & / & $\begin{array}{l}\text { All other } \\
\text { Classes }\end{array}$ & $1 \quad \nabla$ \\
\hline & & $\begin{array}{l}\text { Space Time } \\
\text { Classes }\end{array}$ & E92 \\
\hline
\end{tabular}

Figure 4. Colors and Iconographic Scheme 
These colours and icons are meant to give quick and simple mnemonic clues as to the function of the class or relation within the ontology and how it might relate to the whole.

Thus the user when interacting with the class and relation cards immediately understands what part of the overall ontology they are interacting with and what a particular nexus of classes and relations allows one to say. Furthermore, the colour coding when used on the relationship cards helps learners apprehend the concepts of domain and range as well as quickly sort out appropriate and inappropriate relations for the class that they are working with. In the case where a super-class has sub-classes belonging to more than one high level grouping, this class is assigned multiple colours. This mechanism thus also allows the introduction of the notion of multiple inheritance.

3.3.2 Game Scenario: The basic game materials could potentially be used in any number of scenarios. For the initial game implementation a two stage game scenario was designed with the goal of progressively introducing players to the use and function of the classes and relations in CIDOC CRM. Crucial to the scenario design is a real example from a cultural heritage situation of relevance to the audience at hand. As described above, the context of an interdisciplinary project on the church of Asinou was chosen as the reference case relative to our target audience.

This scenario provided instance cards that would represent potential data units within individual data structures that would need to be translated into CIDOC CRM form. We relied on the players' intimate knowledge of the case study as the background information from which to draw upon in order to put together the data through meaningful relations. In playing the game, the players have in mind to create data structures relevant to their area of research and progressively see how this data could be connected to the research of others.

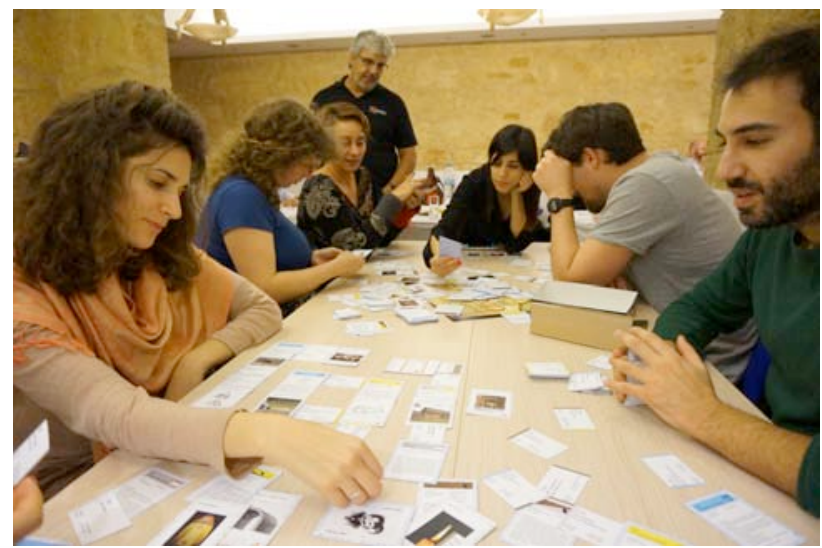

Figure 5. Teams playing the CIDOC CRM Game in a workshop

In the first stage of the game, players are given game boards which have slots available for instance cards and class cards. Players are organized into teams. Working collaboratively is important and useful if possible since it allows players to discuss possible solutions and difficult concepts, helping each other in the process of acquiring the ontology's logical structure. The teams are asked to draw 5 cards at random from the instance card deck. They are then given a set amount of time (adjustable depending on the audience) to match instance cards to class cards for which they would be appropriate instances. This stage of the game familiarizes players with classes, instantiation and IsA hierarchies. One point is given for each correct instantiation under an appropriate class. A variant of the game can create more competition between the teams by allowing a second chance to an opposing team to challenge the mapping. If they can find a class that is more precise to capture the meaning of the instance placed under a class, they can steal the points of the opposite team. This first stage of the game connects the abstract classes to tangible entities in the real world that might be represented in a cultural heritage database. It attempts to give flesh to the abstract classes of the ontology and open players to an appreciation of its versatility for enabling structured discourse over a wide array of phenomena in their domain.

In the second stage of the game, players are challenged to increase their understanding and manipulation of the ontology by filling in the relations between the entities they have indicated as being instances of classes in the previous stage of the game. The game boards already have an empty space for indicating relations and expansion game boards allow the relations to be stretched indefinitely. Using their knowledge of the case study as guide to facts / state of affairs to be modelled, players attempt to relate the instances they have put under classes through appropriate relations. Each correct deployment of a relation card earns the team 2 additional points. In a variant of the game, to increase competition but also to demonstrate the flexibility of graph based representation of data, teams can attempt to hook their class/instances to the class/instances of opposing teams, for which double points can be awarded. This increases the competitive feeling of the game but also physically demonstrates how a web of knowledge can be created using a formal ontology representation.

\subsection{Experience and Analysis}

Running the game experiment in practice proved a learning opportunity both for the target audience and for the game designers in understanding what did and did not work in the game design and the extent of its utility in communicating the ideas and skills relevant to understanding formal ontology and CIDOC CRM. During the running of the game workshops which were held, we observed the players in action, trying to understand what parts of the game facilitated or did not facilitate the appropriation of formal ontology concepts. Furthermore, a questionnaire was distributed at the end of each session seeking feedback on the game from the learner's perspective.

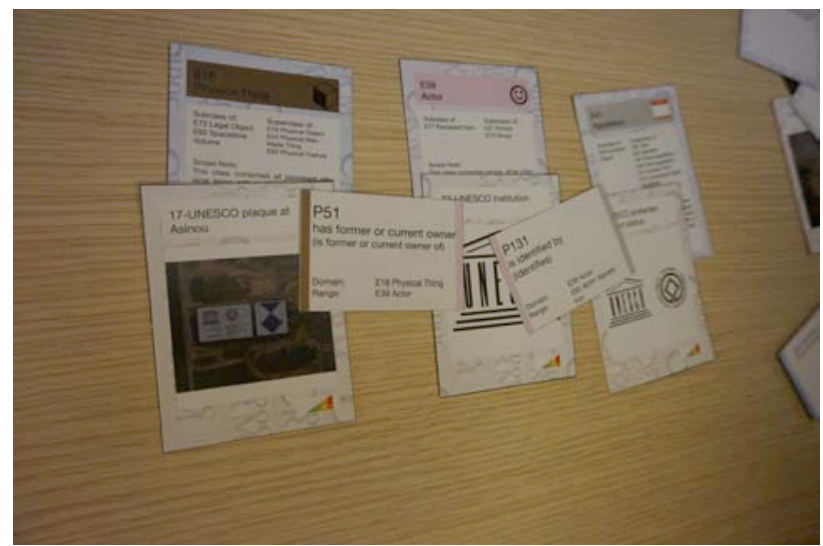

Figure 6. Example of Instance Cards mapped with CRM Cards

According the answers from the questionnaire, reaction from the learning groups was overall highly positive toward the notion of appropriating CIDOC CRM through an interactive game process. The context of team-work allowed the players to 
work together to understand the ontology and its distinctions and then to begin to understand the formal mechanisms for relating information. Working directly with the ontology through a physical format offered a direct way for the learners to engage with the concepts from the very basic first steps. The learners also strongly appreciated the case study scenario, using familiar data, which was both easy to understand and also entertaining. Interestingly, the competitive context of a game was also a positive motivator to the learners who were encouraged to improve their understanding in order to beat competing teams.

Analyzing the learners' interaction with the game, it was clear to the trainers that the game engaged the participants in genuine ontology and modelling discussions, allowing them to directly face the questions and the concepts related to CIDOC CRM. The learners were pushed to engage with the definitions of classes and relations and to turn to the ontology documentation in order to pursue the gaming process. The overall results were highly encouraging both for the level of enthusiasm generated and for the ability of the game context to facilitate ontology/CIDOC CRM knowledge transfer.

That being said, the game design is not considered to be complete, but the first in an iterative series of versions to be designed based on feedback from and observation of the learners during the gaming sessions. In order to explore the potentials of the game and to address difficulties of understanding/presentation of the game, a series of additional versions were made since the initial implementation. Particularly, a scenario for book and paper conservation ${ }^{1}$ was created and tested, which retooled the game, for a specific context, changing the game mechanism to offer more specific cards and a more rapid pace of play. Further, an effort was made to explore how the game could be used to introduce CIDOC CRM extensions. Thus a version of the game ${ }^{2}$ was designed with a case study looking at archaeological excavation records referencing built remains. This version allows the exploration of the concepts and relations deployed in the CRMArchaeo and CRMBa, extensions to the core ontology for the documentation of excavation practice and building archaeology respectively.

\section{CONCLUSION}

The CIDOC CRM as a formal ontology has enormous potential for enabling the integration of datasets in the cultural heritage community but faces a roadblock to widespread adoption in the lack of tools for an easy, popular dissemination of the standard. The CIDOC CRM game was designed as a potential solution to introducing this formal ontology to domain experts with no specialization in information science. The effort proved both successful at offering an entry point to the concepts of CIDOC $\mathrm{CRM}$ and to creating a positive learning environment for exploring them. As opposed to a lecture format, the introduction of the CIDOC CRM through a hands-on activity allows learners to engage with the material by active visual, tactile and oral means. The game is fun, hands on, interactive and linked to known examples. It allows the concrete demonstration of the meaning of ideas such as instantiation and ontological relations, showing concretely what a knowledge graph is. The game has the potential to form an important tool in providing a concrete path into a functional understanding of CIDOC CRM. For the games wider use, the elaboration of further scenarios to explore CIDOC CRM and its extensions would be required. This could

\footnotetext{
${ }^{1}$ Athanasis Velios, University of the Arts, London.

2 Paola Ronzino and Achille Felicetti, PIN, Prato.
}

include both the elaboration of well known case studies to be used at a general level as well as specific scenarios generated to introduce the concepts to target audiences needing to appropriate CIDOC CRM for a specific need. Both disciplinary and cultural interest should be taken into account in the elaboration of these scenarios. Furthermore, the card format of the game allows for virtually unlimited elaboration of new game design for the manner in which to play with the cards. The game of course stands not as an end in itself, but should be considered as a tool to introduce concepts, forming part of an overall educational pipeline to bring learners from no knowledge of CRM to the capacity to both understand and apply CRM in the practical scenario of real data integration activities. Finally, it is worth noting that the method of the game seems suitable for the teaching of formal ontology concepts in general and need not be restricted to any particular ontology.

\section{ACKNOWLEDGEMENTS}

The present work has been supported by the Marie-Curie action ITN-DCH and the ARIADNE project. The ITN-DCH project has received funding from the European Union's Seventh Framework Programme for research, technological development and demonstration under grant agreement no 608013. The ARIADNE project was funded by the European Commission (grant 313193) under the FP7 INFRA-2012-1.1.3 call. The authors' opinion do not necessarily reflect those of the European Commission.

\section{REFERENCES}

Cooper, A., 1999. The Inmates are Running the Asylum: Why High-Tech Products Drive Us Crazy and How 2 to Restore the Sanity. SAMS.

Doerr, M., and Crofts, N., 1999. Electronic Esperanto - The Role of the CIDOC Reference Model. Proceedings of the ICHIM '99, Washington DC, 22-26 September 1999.

Doerr, M., 2003. The CIDOC conceptual reference module: an ontological approach to semantic interoperability of metadata. AI magazine 24: 75.

Doerr, M., Ore, C. E., \& Stead, S., 2007. The CIDOC conceptual reference model: a new standard for knowledge sharing. Tutorials, posters, panels and industrial contributions at the 26th international conference on conceptual modeling, Australian Computer Society Inc., Vol. 83, pp. 51-56.

Doerr, M. and Iorizzo, D., 2008. The Dream of a Global Knowledge Network: A New Approach. J. Comput. Cult. Herit., 1 (5), pp. 1-23.

Doerr, M., 2009. Ontologies for cultural heritage. Handbook on Ontologies. Springer, pp. 463-486.

Guarino, N., 1998. Formal ontology and information systems. Proceedings of the International Conference on Formal Ontology in Information Systems, Vol. 98, pp. 81-97.

Oldman, D., Doerr, M., de Jong, G., Norton, B. and Wikman, T., 2014. Realizing Lessons of the Last 20 Years: A Manifesto for Data Provisioning and Aggregation Services for the Digital Humanities System. D-Lib Magazine, 20(7/8). https://doi.org/10.1045/july2014-oldman 
Smith, B. and Welty, C., 2001. Ontology-towards a New Synthesis. Proceedings of the International Conference on Formal Ontology in Information Systems, New York, USA, ACM, pp .3-.9. https://doi.org/10.1145/505168.505201

Smith, B. (2003). Ontology. Blackwell Guide to the Philosophy of Computing and Information, Oxford: Blackwell, pp. 155166.

Sure, Y., Staab, S., and Studer, R., 2009. Ontology engineering methodology. Handbook on ontologies, Springer, pp. 135-152. 